\title{
Alteration of Epigenetic Profile in Human Hepatocellular Carcinoma and Its Clinical Implications
}

\author{
Naoshi Nishida Masatoshi Kudo \\ Department of Gastroenterology and Hepatology, Kinki University Faculty of Medicine, Osaka, Japan
}

\author{
Key Words \\ DNA methylation · Etiology $\cdot$ Hepatocellular carcinoma $\cdot$ Transcription
}

\begin{abstract}
Hepatocellular carcinoma (HCC) is a common cancer worldwide and develops against a background of chronic liver damage. A variety of HCC-related genes are known to be altered by genetic and epigenetic mechanisms. Therefore, information regarding alteration of the genetic and epigenetic profiles in HCC is essential for understanding the biology of this type of tumor. Methylation at CPG sites in gene promoters is known to affect the transcription of the corresponding genes. Abnormal regional hypermethylation is observed in the $5^{\prime}$ region of several tumor suppressor genes (TSGs) in HCC, and this hypermethylation may promote carcinogenesis through the transcriptional inactivation of downstream TSGs. The DNA damage induced by oxidation is a trigger of abnormal DNA methylation and inactivation of TSGs through recruitment of the polycomb repressive complex to the promoter sequence. Thus, oxidative stress may be responsible for the emergence of HCC from chronic hepatitis and liver cirrhosis through the epigenetic alteration of TSGs. There have been several attempts to apply epigenetic information to the diagnosis and treatment of HCC. The predictive value of selected methylation events on survival in HCC patients has been reported, and the methylation profile of background liver could be associated with recurrence-free survival of HCC patients who have undergone hepatectomy. Another study detected methylated DNA from HCC cells in serum, and the circulating tumor DNA was regarded as a potential tumor marker. In addition, several trials of HCC therapy have targeted the epigenetic machinery and were based upon comprehensive analyses of DNA methylation of this type of tumor. Here, we present an overview of research regarding DNA methylation status in human HCC and describe the clinical application of epigenetic information to HCC. Copyright $\odot 2014$ S. Karger AG, Basel
\end{abstract}




\section{Introduction}

The methylation status at $\mathrm{CpG}$ sites in gene promoters, which are generally located within CpG islands, is closely associated with transcription of the corresponding genes. In cancerous tissues, abnormal regional hypermethylation observed in the $5^{\prime}$ region of several tumor suppressor genes (TSGs) is thought to promote carcinogenesis through the transcriptional inactivation of downstream TSGs. On the other hand, global DNA hypomethylation is another characteristic of cancer and is thought to induce activation of transposons, oncogenes, and chromosomal alterations, thereby contributing to carcinogenesis [1, 2].

In human hepatocellular carcinoma (HCC), a variety of TSGs have shown abnormal hypermethylation in their promoter regions and down-regulation of their transcripts, suggesting the potential role of inactivation of these TSGs in hepatocarcinogenesis [3]. A relationship between the extent of abnormal chromosomal regions and the degree of DNA hypomethylation at repetitive DNA sequences has also been reported. This evidence supports the idea that global DNA hypomethylation could induce chromosomal instability and contribute to hepatocarcinogenesis [4]. Results from another study involving human HCC samples revealed an association between down-regulation of microRNA (miR) and abnormal methylation of the host gene [5]. This review gives a synopsis of comprehensive analyses of DNA methylation in human HCC and further focuses on the clinical application of epigenetic information, such as alteration of the histone code or DNA methylations in HCC.

\section{Origin of Epigenetic Alteration in Human HCC}

Although the methylation levels in TSG promoters are much higher in HCC than in noncancerous liver, abnormal methylation has also been observed in the promoters of TSGs in non-cancerous liver of HCC patients. Additionally, several studies have confirmed that the methylation levels of non-cancerous liver show a progression from chronic hepatitis to liver cirrhosis, in parallel with the increase in risk for the development of HCC. Viral infection, chronic inflammation, and oxidative stress can induce abnormal DNA methylation in affected hepatocytes. Thus, chronic hepatitis and liver cirrhosis may be the background conditions that lead to HCC emergence via abnormal methylation patterns that result in transcriptional inactivation of TSGs.

Several studies have reported a mechanistic link between epigenetic instability and the oxidative stress induced by inflammation. HCC cells are known to frequently carry abnormally methylated E-cadherin genes. Lim et al. reported that reactive oxygen species (ROS) could induce the expression of Snail, a repressive transcription factor of E-cadherin, through activation of the PI3K-Akt pathway and induce hypermethylation of the E-cadherin promoter by recruiting histone deacetylase 1 (HDAC1) and DNA methyltransferase 1 (DNMT1) [6]. They also showed a correlation between ROS induction, E-cadherin down-regulation, Snail up-regulation, and the E-cadherin promoter methylation in HCC tissues.

$\mathrm{O}^{\prime} \mathrm{Hagan}$ et al. demonstrated that inducing cellular oxidative stress in a colorectal cancer cell line using hydrogen peroxide $\left(\mathrm{H}_{2} \mathrm{O}_{2}\right)$ treatment recruits DNMT1 to damaged chromatin and causes relocalization of DNMTs and other members of the polycomb repressive complex 4 from non-GC-rich to GC-rich areas [7]. They also showed an increase in repressive histone modification, such as the trimethylation of lysine 27 of histone H3 (H3K27), and a decrease in active histone modification, such as the trimethylation of H3K4 and the acetylation of H4K16, on DNA sites showing oxidative damage. Using an HCC cell line and fetal liver cells, we also demonstrated that $\mathrm{H}_{2} \mathrm{O}_{2}$ treatment increases oxidative DNA damage on the promoters of multiple cancer-related genes that are known to show abnormal DNA methylation in 
many types of cancers. We confirmed that $\mathrm{H}_{2} \mathrm{O}_{2}$ treatment alters histone modifications on these promoters from active to repressive patterns predominantly at damaged DNA sites [8].

Furthermore, using mice with humanized livers, Okamoto et al. reported that hepatitis B virus (HBV) or hepatitis $\mathrm{C}$ virus (HCV) infection could induce genome-wide, time-dependent changes in DNA methylation. Inhibition of natural killer cells or administration of neutralizing antibody of interferon- $\gamma$ could inhibit methylation changes in the infected mice [9]. These results strongly support the idea that chronic inflammation and viral infection can cause a disturbance in DNA methylation status through the immune response as well as by the induction of ROS.

Increasing evidence suggests that non-alcoholic fatty liver disease (NAFLD) may be a risk factor for the emergence of HCC, and oxidative stress is believed to be a causal factor in NAFLD progression. From this observation, more methylation changes are likely to accumulate in the liver of patients with advanced NAFLD than in those with mild NAFLD. A recent report described many differently methylated CpGs between liver samples from patients with advanced and mild NAFLD, suggesting that epigenetic alteration also plays a role in NAFLDrelated HCC emergence [10]. In that study, methylation levels were compared between mild and advanced NAFLD for more than 450,000 CpG sites, and about 70,000 CpG sites revealed methylation differences. Methylation correlated with gene transcript levels for $7 \%$ of differentially methylated $\mathrm{CpG}$ sites, indicating that these methylations affect expression of the corresponding genes. Tissue repair genes were hypomethylated and overexpressed, whereas genes in certain metabolic pathways were hypermethylated and underexpressed in advanced NAFLD [10].

\section{Methylation Events Drive Hepatocarcinogenesis}

Alteration of DNA methylation takes place in various promoters, gene bodies, intragenic regions, and repetitive DNA sequences. However, the importance of individual methylation changes to carcinogenesis is mostly unknown. Gao et al. determined and classified the pattern of methylation progression in 6,458 CpG sites in normal liver, non-cancerous liver of HCC patients, and HCC tissues. Some methylation events were observed in HCC tissues but were not found in non-cancerous liver and normal liver [11], suggesting the importance of this type of methylation in hepatocarcinogenesis. To determine the methylation events that might contribute to the emergence of HCC, we analyzed the methylation profiles of early HCC (eHCC), which is defined as HCC with hypovascular tumors $<2 \mathrm{~cm}$, and compared them with those of non-cancerous liver and progressive HCC.

We also successfully classified methylation events into three patterns: methylation events showing prominent differences between non-cancerous liver and eHCC, methylation events showing gradual increase according to tumor progression, and methylation that could be detected in advanced tumors only [12]. It is noteworthy that the elevated levels of methylation detected in eHCC were also observed in non-cancerous liver, although the levels were much lower than those in HCC tissue [13]. Interestingly, the number of methylated TSGs in livers infected with chronic hepatitis $\mathrm{C}$ was significantly associated with the time taken for HCC to occur in patients with no history of HCC [12].

Therefore, we concluded that these methylation events might act as a driver that could accelerate hepatocarcinogenesis. On the other hand, although a number of methylation events were observed in advanced HCC, the majority of them were quantitatively low level and might be "passive" methylation events that emerge as a consequence of carcinogenesis, rather than being the drivers of carcinogenesis. In addition, we observed methylation events in normal 
livers among the elderly [13]. It is conceivable that ROS induced by normal metabolic processes might cause abnormal DNA methylation in hepatocytes in an age-dependent manner, thereby promoting HCC emergence in livers without severe fibrosis. Recently, HCC has been observed in livers without advanced fibrosis, especially in elderly patients. This evidence also supports the idea that methylation events that are responsible for hepatocarcinogenesis exist in normal livers among the elderly as well as in livers infected with chronic hepatitis.

\section{Differently Methylated Regions between HCC and Background Liver}

As described above, in normal cells, the cytosine of CpG sites within TSG promoters and CpG-rich regions are generally unmethylated, whereas in other DNA regions, such as repetitive DNA sequences, these sites are densely methylated. However, TSG promoters showed regional hypermethylation in cancer cells. Irizarry et al. showed that, in colon cancer, most cancer related-alterations of DNA methylation did not take place in CpG islands, but in sequences up to $2 \mathrm{~kb}$ away; such regions were termed "CpG island shores" [14]. It was also reported that regions showing different methylation patterns between cancer and normal epithelial cells [cancer-specific differentially methylated regions (C-DMRs)] showed considerable overlap with those showing different methylation patterns among different tissues [tissue-specific differentially methylated regions]. Therefore, it is possible that the majority of methylation alterations found in cancer occur at $\mathrm{CpG}$ sites where epigenetic changes take place during tissue differentiation, and that some epigenetic alterations found in cancer might cause stem cell-like phenotypes, such as pluripotency. On the other hand, Ammerpohl et al. reported that several genes that showed abnormal methylation in human HCC were the targets of polycomb repressive complex 2 in embryonic stem cells $[15,16]$. Polycomband trithorax-group proteins are known to participate in the modulation of histones and the alteration of gene expression patterns during cell differentiation [1]. Given that repressive histone modulation is associated with methylation of the corresponding genes, some methylation events could induce undifferentiated phenotypes through the transcriptional inactivation of genes necessary for cell differentiation.

\section{Methylation Profile in HCC}

Several studies have reported comprehensive analyses of the DNA methylation profile of HCC (table 1). A recent study analyzed more than 450,000 CpG sites and showed the methylation profile of HCC throughout the whole genome. According to the report, $60.1 \%$ of the CpGs that had undergone hypermethylation in HCC were located in CpG islands, $21.6 \%$ were in CpG shores, and 3.6\% were in CpG shelves, which is defined as the region just outside CpG shores [17]. In contrast, the majority of hypomethylated CpGs in HCC were located away from CpG islands. Another group also reported that hypermethylated CpGs were mainly distributed close to transcription start sites, and the majority of hypomethylated CpGs were observed within $3^{\prime}$ regions of genes and intragenic regions [18].

Additionally, although hypomethylated CpGs were observed more frequently than hypermethylated TSGs were, the C-DMRs of HCC were observed more frequently in hypermethylated than in hypomethylated regions [17]. Because regional alteration of methylation may affect gene transcription more strongly than alteration of individual $\mathrm{CpG}$ methylation does, DNA hypermethylation may play a role in HCC emergence predominantly through the transcriptional inactivation of TSGs; however, DNA hypomethylation may contribute to car- 


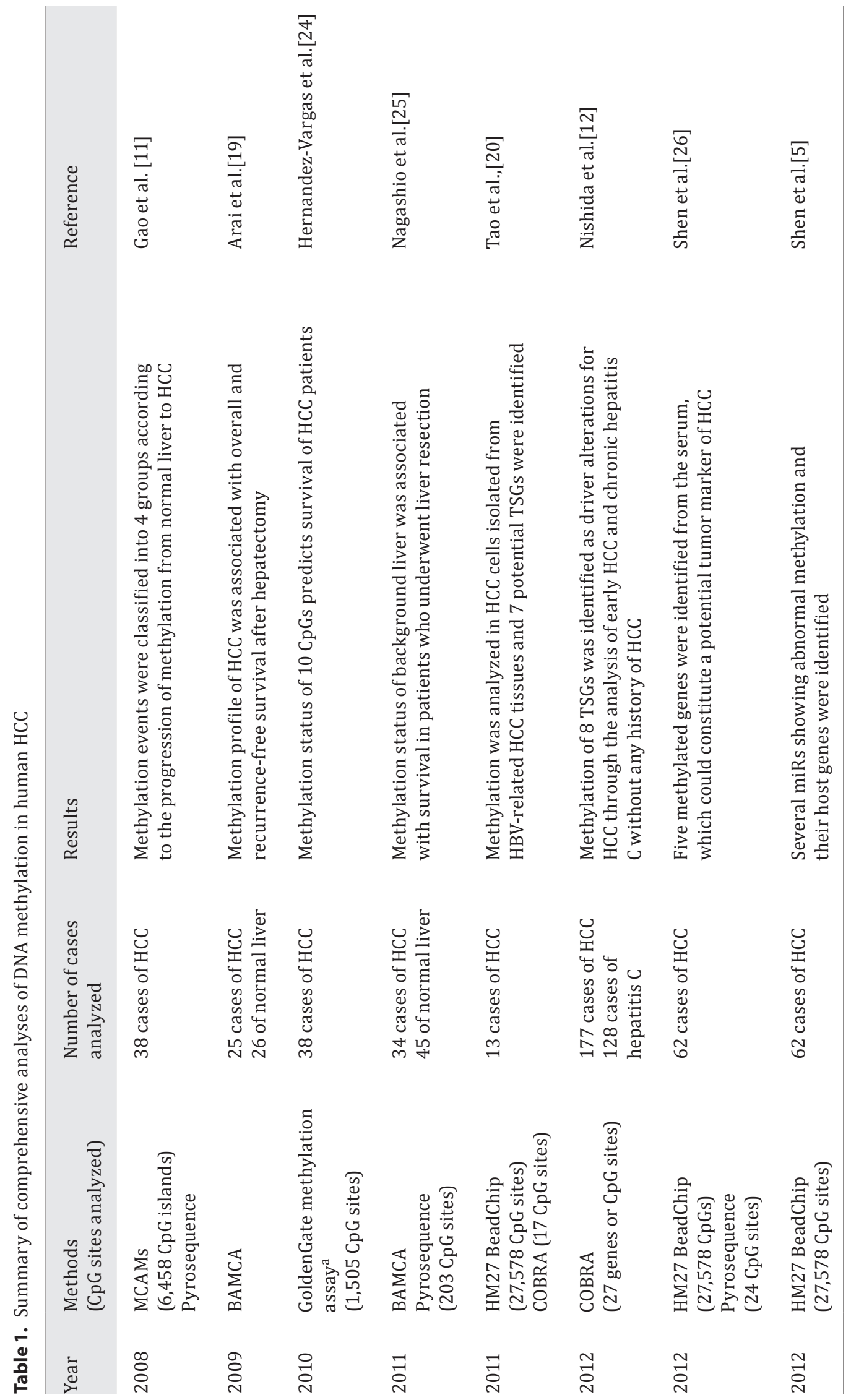




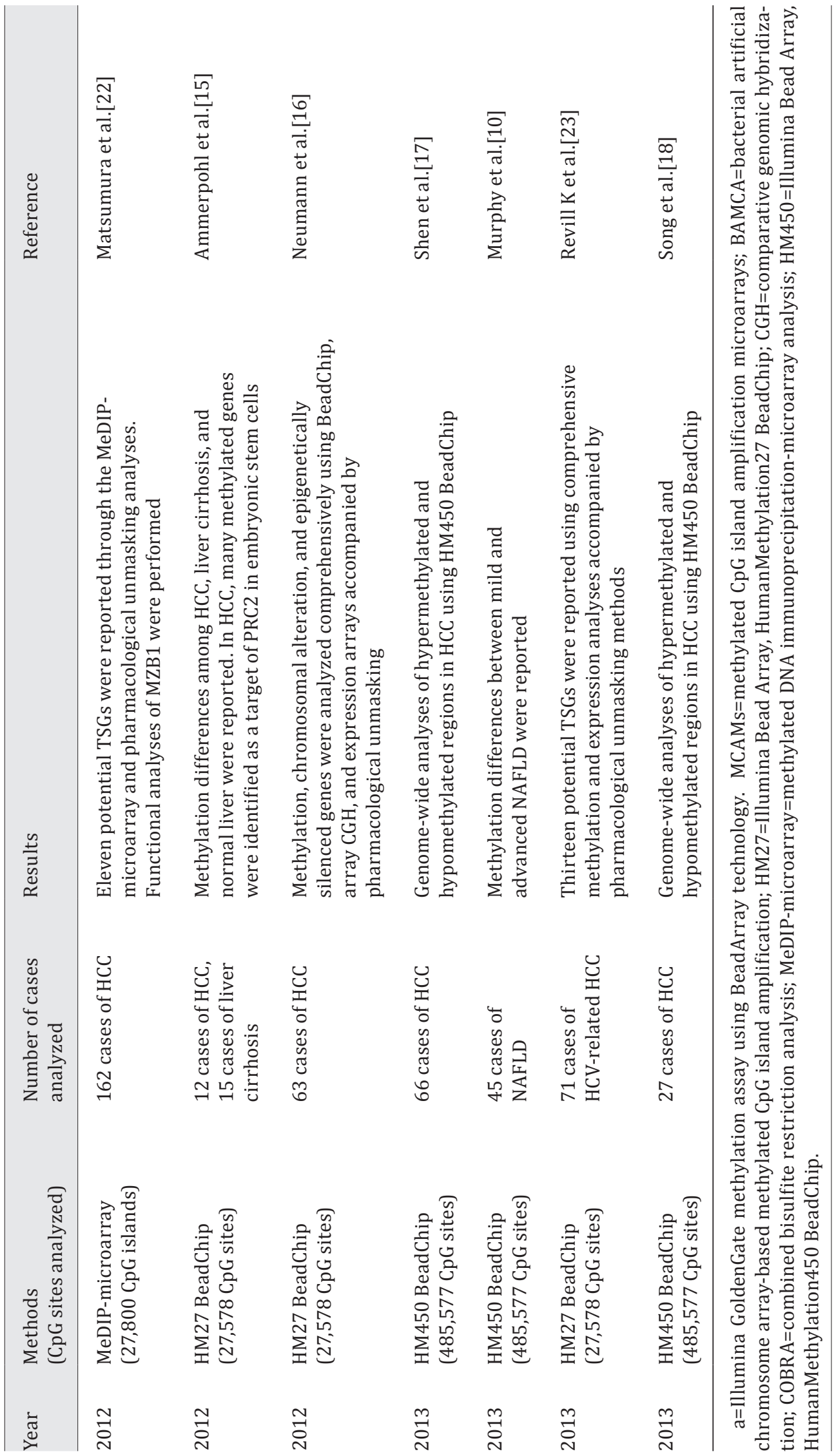


cinogenesis by additional mechanisms, such as induction of chromosomal instability. We also reported that the global DNA hypomethylation level was closely associated with the extent of the altered chromosomal region, and this association was independent of the tumor stage [4].

\section{DNA Methylation and Etiology of HCC}

Results from a previous study identified several methylation events that were associated with the etiology of HCC and background liver status: 75 CpGs showed differences in methylation patterns between livers of males and females, methylation at $228 \mathrm{CpGs}$ was associated with HCV infection, and methylation at 17,207 CpGs showed different levels between cirrhotic and non-cirrhotic livers [17]. Among the 228 CpGs associated with HCV infection, methylation events at $56 \mathrm{CpGs}$ also showed association with the presence of liver cirrhosis. The considerable overlap of HCV-associated and cirrhosis-associated methylation events suggested that HCC may be more commonly observed in livers that are infected with HCV and are cirrhotic. Additionally, methylation of TSG promoters was more prevalent in liver cirrhosis than in chronic hepatitis [11], and the methylation status of TSG promoters in background liver reflected the methylation profile of matched HCC tissues [19]. For example, HCV-related HCC, which frequently arises from cirrhotic liver, is known to carry many methylated TSGs, and methylation of TSG promoters is more prominent in HCV-positive than in HCV-negative liver tissues [13]. Therefore, it is possible that the duration of inflammation is related to the progression of liver fibrosis and to TSG methylation, which is more common in chronic hepatitis C.

Other studies analyzed the alteration of cancer-related pathways that might be affected by methylation-associated inactivation of TSGs. Shen et al. classified genes showing abnormal methylation and reported several pathways that involved genes showing increased or decreased methylation, such as G-protein, endothelin, PI3-K, interleukin, inflammatory cytokines, and insulin/growth factor signaling pathways [5]. Tao et al. analyzed DNA methylation in single isolated HCC cells, thereby eliminating the noise caused by the methylation of nonparenchymal cells, and reported that DNA methylation could lead to abnormal cellular function in gap junctions, calcium signaling, cell adhesion, and apoptosis [20]. However, because a variety of genes showed abnormal methylation in HCC, it was difficult to evaluate which methylation events might be responsible for carcinogenesis.

\section{Newly Discovered TSGs Showed Abnormal Methylation and Transcriptional Inactivation}

As described above, it is sometimes difficult to tell "driver" methylation events for HCC from "passive" methylation events that are merely consequences of carcinogenesis. Therefore, the effects of each methylation event need to be confirmed. A recent study isolated HBVrelated HCC cells from cancerous tissues and performed comprehensive methylation analyses. Seven new genes were identified with abnormal methylation in HCC: WNK2, EMILIN2, TRIM58, GRASP, TM6SF1, HIST1H4F, and TLX3 [20]. After treatment of these cancer cells with DNMT inhibitors, transcriptional reactivation was induced in these genes, suggesting that they were inactivated in HCC as a result of abnormal methylation. Gao et al. identified seven hypermethylated genes in HCC: miR-219, MMP-14, RASSF1A, TBX4, GNA14, CDKN2A, and CCNA1. They also confirmed re-expression by a pharmacological unmasking method in which an HCC cell line was treated with DNMT inhibitor 5-aza-deoxycytosine [11]. 
Neumann et al. conducted a study that involved the combination of comprehensive methylation and expression analyses before and after pharmacological unmasking. They determined the comprehensive methylation profile, chromosomal status, and epigenetically silenced genes of human HCC using HumanMethylation450 BeadArray (Illumina), array comparative genomic hybridization, and pharmacological unmasking expression array analysis. They reported three candidate TSGs (PER3, PROZ, and IGFALS) that showed abnormal methylation in HCC, loss of corresponding chromosomal regions, and re-expression after pharmacological unmasking [16]. Among these candidate TSGs, the functions of PER3 and IGFALS have already been reported, and they were shown to be potential TSGs of HCC [21]. Matsumura et al. also performed $\mathrm{CpG}$ island microarray analysis accompanied with pharmacological unmasking and reported MZB1 as a new TSG of HCC [22]; the down-regulation of MZB1 was reportedly associated with survival of HCC patients. Revill et al. performed promoter methylation profiles using HumanMethylation27 BeadChip and expression array analysis combined with pharmacological unmasking and found 13 candidate TSGs [23]. That study showed that transfection of SMPD3 and NEFH led to growth inhibition in HCC cell lines, and knockdown of these genes by small interfering RNA induced tumor formation and invasiveness in nude mice, indicating that these were potential TSGs. The expression of SMPD3 was reportedly associated with recurrence-free survival after curative resection of HCC [23].

The transcription of non-coding RNA, such as miR and long non-coding RNA, is also known to be regulated by DNA methylation. The role of miR in carcinogenesis is particularly well recognized. Coding regions of $\mathrm{miR}$ are generally located within the intron of host genes, and abnormal methylation of host genes leads to transcriptional inactivation of miR. Several DNA methylations are reportedly related to the down-regulation of the corresponding $\mathrm{miR}$. Recently, comprehensive methylation analyses identified hypermethylation of miR host genes, and the role of inactivation of each miR on hepatocarcinogenesis was analyzed [5]. The abnormal methylation of host gene HOXB4 was shown to induce activation of the NF- $\mathrm{BB}$ signaling pathway through transcriptional inactivation of miR-10a [5].

\section{Epigenetic Information and Treatment of HCC}

Because DNA methylation affects the phenotype mainly through expression of the corresponding genes, methylation profiles could reflect the biological characteristics of HCC if "passive methylation" could be eliminated appropriately. Several reports have shown the predictive value of selected methylation events on survival $[19,24]$. The methylation profile of background liver may also be associated with the recurrence-free survival of HCC patients who undergo hepatectomy [25]. We also reported a prominent relationship between the number of methylated TSGs and the time to HCC occurrence by analyzing chronic hepatitis C patients without prior history of HCC [12]. Therefore, DNA methylation is crucial for HCC emergence and may be observed at high levels in livers infected with chronic hepatitis that have a high risk of hepatocarcinogenesis. Another study reported the presence of methylated DNA from HCC cells in serum and described the circulating tumor DNA as a potential tumor marker [26].

Recent HCC therapy trials have targeted the epigenetic machinery based on the comprehensive analyses of DNA methylation profiles of this type of tumor. Several novel moleculartargeted drugs have been developed using specific cancer mutation profiles [27]. However, the mutation profile of HCC is heterogeneous [28], and the frequencies of mutations in specific genes are relatively low. Therefore, it might be difficult to target individually altered genes for HCC therapy [3, 29]. 
On the other hand, a variety of TSGs showed abnormal DNA methylation in HCC [3] and altered epigenetic codes, including abnormal DNA methylation, could be reversed by using DNMT or HDAC inhibitors. For example, DNMT inhibitors induce the expression of inactivated TSGs through removal of promoter methylation, resulting in enhanced antitumor action. Two examples of this type of drug, 5-azacytidine and 5-aza-2'-deoxycytidine (decitabine), have already been approved for treatment of myelodysplastic syndrome and acute myelocytic leukemia [30,31]. However, DNMT inhibitors have proven insufficient for the treatment of solid tumors in contrast to their encouraging activity against hematological malignancies [32]. Several kinds of cancers exhibit overexpression of HDACs, and transcriptional inactivation of growth-inhibitory and apoptosis-related genes have been observed through the abnormal deacetylation of histone tails. Several clinical trials involving the use of HDAC inhibitors, such as vorinostat for treatment of cutaneous T cell lymphoma, are currently ongoing [33].

Epigenetic therapy that modulates the epigenetic machinery could induce the alteration of cancer phenotypes through the rewriting of "abnormal" epigenetic codes; this would represent a distinctive type of therapy that is very different from conventional anti-cancer therapy. For example, cancer cells showing stem cell-like phenotypes are often resistant to conventional chemotherapy as well as to other molecular targeting agents [34]. However, rewriting the altered epigenetic code might induce differentiation in cancer cells, which could induce sensitivity to several types of chemotherapy. Additionally, because epigenetic therapy should be effective even after administration of the active agent has been discontinued, it could be effective in combination with other chemotherapeutic agents, either by administration at the beginning of, or before, the other chemotherapy. So far, no epigenetic therapy has proven effective against human HCC, although a recent report suggested the effectiveness of HDAC inhibitor panobinostat in a mouse xenograft model of HCC in combination with sorafenib [35]. Overexpression of HDAC has been reported in HCC [36]; thus, modulation of the epigenetic machinery should prove a promising approach for treating this type of malignancy.

\section{Conclusion}

Understanding the alteration of genetic and epigenetic codes is essential to predict the biological behavior and effectiveness of cancer therapy, because both codes represent important bioinformation regarding cancer [2]. The genetic code determines protein structure and quality, whereas the epigenetic code determines the quantity of protein produced. However, normal cells may have epigenetic codes that have been altered as a result of different physiological processes. As a result, it is more difficult to narrow down cancer-specific alterations in DNA methylation than those in gene mutation. Nonetheless, recent technological advancements such as BeadArray technology and next-generation sequencers allow us to perform comprehensive genome-wide analyses from a tiny amount of DNA. Therefore, it is possible to target a larger number of small, early stage lesions for comprehensive analyses [37]. It is anticipated that rapid technological progress and understanding of the alteration of the genetic and epigenetic codes will provide essential information for developing novel diagnostic approaches and therapies for HCC in the near feature.

\section{References}

1 Baylin SB, Jones PA: A decade of exploring the cancer epigenome - biological and translational implications. Nat Rev Cancer 2011;11:726-734.

2 You JS, Jones PA: Cancer genetics and epigenetics: two sides of the same coin? Cancer Cell 2012;22:9-20. 
3 Nishida N, Goel A: Genetic and epigenetic signatures in human hepatocellular carcinoma: a systematic review. Curr Genomics 2011;12:130-137.

4 Nishida N, Kudo M, Nishimura T, Arizumi T, Takita M, Kitai S, Yada N, Hagiwara S, Inoue T, Minami Y, Ueshima K, Sakurai T, Yokomichi N, Nagasaka T, Goel A: Unique association between global DNA hypomethylation and chromosomal alterations in human hepatocellular carcinoma. PLoS ONE 2013;8:e72312.

-5 Shen J, Wang S, Zhang YJ, Kappil MA, Chen Wu H, Kibriya MG, Wang Q, Jasmine F, Ahsan H, Lee PH, Yu MW, Chen CJ, Santella RM: Genome-wide aberrant DNA methylation of microRNA host genes in hepatocellular carcinoma. Epigenetics 2012;7:1230-1237.

6 6 Lim SO, Gu JM, Kim MS, Kim HS, Park YN, Park CK, Cho JW, Park YM, Jung G: Epigenetic changes induced by reactive oxygen species in hepatocellular carcinoma: methylation of the E-cadherin promoter. Gastroenterology 2008;135:2128-2140, e1-e8.

7 O'Hagan HM, Wang W, Sen S, Destefano Shields C, Lee SS, Zhang YW, Clements EG, Cai Y, Van Neste L, Easwaran H, Casero RA, Sears CL, Baylin SB: Oxidative damage targets complexes containing DNA methyltransferases, SIRT1, and polycomb members to promoter CpG Islands. Cancer Cell 2011;20:606-619.

-8 Nishida N, Arizumi T, Takita M, Kitai S, Yada N, Hagiwara S, Inoue T, Minami Y, Ueshima K, Sakurai T, Kudo M: Reactive oxygen species induce epigenetic instability through the formation of 8-hydroxydeoxyguanosine in human hepatocarcinogenesis. Dig Dis 2013;31:459-466.

>9 Okamoto Y, Shinjo K, Shimizu Y, Sano T, Yamao K, Gao W, Fujii M, Osada H, Sekido Y, Murakami S, Tanaka Y, Joh T, Sato S, Takahashi S, Wakita T, Zhu J, Issa JP, Kondo Y: Hepatitis virus infection affects DNA methylation in mice with humanized livers. Gastroenterology 2014;146:562-572.

-10 Murphy SK, Yang H, Moylan CA, Pang H, Dellinger A, Abdelmalek MF, Garrett ME, Ashley-Koch A, Suzuki A, Tillmann HL, Hauser MA, Diehl AM: Relationship between methylome and transcriptome in patients with nonalcoholic fatty liver disease. Gastroenterology 2013;145:1076-1087.

11 Gao W, Kondo Y, Shen L, Shimizu Y, Sano T, Yamao K, Natsume A, Goto Y, Ito M, Murakami H, Osada H, Zhang J, Issa JP, Sekido Y: Variable DNA methylation patterns associated with progression of disease in hepatocellular carcinomas. Carcinogenesis 2008;29:1901-1910.

12 Nishida N, Kudo M, Nagasaka T, Ikai I, Goel A: Characteristic patterns of altered DNA methylation predict emergence of human hepatocellular carcinoma. Hepatology 2012;56:994-1003.

-13 Nishida N, Nagasaka T, Nishimura T, Ikai I, Boland CR, Goel A: Aberrant methylation of multiple tumor suppressor genes in aging liver, chronic hepatitis, and hepatocellular carcinoma. Hepatology 2008;47:908918.

14 Irizarry RA, Ladd-Acosta C, Wen B, Wu Z, Montano C, Onyango P, Cui H, Gabo K, Rongione M, Webster M, Ji H, Potash JB, Sabunciyan S, Feinberg AP: The human colon cancer methylome shows similar hypo- and hypermethylation at conserved tissue-specific CpG island shores. Nat Genet 2009;41:178-186.

-15 Ammerpohl O, Pratschke J, Schafmayer C, Haake A, Faber W, von Kampen O, Brosch M, Sipos B, von Schönfels W, Balschun K, Röcken C, Arlt A, Schniewind B, Grauholm J, Kalthoff H, Neuhaus P, Stickel F, Schreiber S, Becker T, Siebert R, Hampe J: Distinct DNA methylation patterns in cirrhotic liver and hepatocellular carcinoma. Int J Cancer 2012;130:1319-1328.

-16 Neumann O, Kesselmeier M, Geffers R, Pellegrino R, Radlwimmer B, Hoffmann K, Ehemann V, Schemmer $\mathrm{P}$, Schirmacher P, Lorenzo Bermejo J, Longerich T: Methylome analysis and integrative profiling of human HCCs identify novel protumorigenic factors. Hepatology 2012;56:1817-1827.

17 Shen J, Wang S, Zhang YJ, Wu HC, Kibriya MG, Jasmine F, Ahsan H, Wu DP, Siegel AB, Remotti H, Santella RM: Exploring genome-wide DNA methylation profiles altered in hepatocellular carcinoma using Infinium HumanMethylation 450 BeadChips. Epigenetics 2013;8:34-43.

18 Song MA, Tiirikainen M, Kwee S, Okimoto G, Yu H, Wong LL: Elucidating the landscape of aberrant DNA methylation in hepatocellular carcinoma. PLoS ONE 2013;8:e55761.

19 Arai E, Ushijima S, Gotoh M, Ojima H, Kosuge T, Hosoda F, Shibata T, Kondo T, Yokoi S, Imoto I, Inazawa J, Hirohashi S, Kanai Y: Genome-wide DNA methylation profiles in liver tissue at the precancerous stage and in hepatocellular carcinoma. Int J Cancer 2009;125:2854-2862.

-20 Tao R, Li J, Xin J, Wu J, Guo J, Zhang L, Jiang L, Zhang W, Yang Z, Li L: Methylation profile of single hepatocytes derived from hepatitis B virus-related hepatocellular carcinoma. PLoS ONE 2011;6:e19862.

-21 Lin YM, Chang JH, Yeh KT, Yang MY, Liu TC, Lin SF, Su WW, Chang JG: Disturbance of circadian gene expression in hepatocellular carcinoma. Mol Carcinog 2008;47:925-933.

22 Matsumura S, Imoto I, Kozaki K, Matsui T, Muramatsu T, Furuta M, Tanaka S, Sakamoto M, Arii S, Inazawa J: Integrative array-based approach identifies MZB1 as a frequently methylated putative tumor suppressor in hepatocellular carcinoma. Clin Cancer Res 2012;18:3541-3551.

23 Revill K, Wang T, Lachenmayer A, Kojima K, Harrington A, Li J, Hoshida Y, Llovet JM, Powers S: Genomewide methylation analysis and epigenetic unmasking identify tumor suppressor genes in hepatocellular carcinoma. Gastroenterology 2013;145:1424-1435, e1-e25.

24 Hernandez-Vargas H, Lambert MP, Le Calvez-Kelm F, Gouysse G, McKay-Chopin S, Tavtigian SV, Scoazec JY, Herceg Z: Hepatocellular carcinoma displays distinct DNA methylation signatures with potential as clinical predictors. PLoS ONE 2010;5:e9749.

25 Nagashio R, Arai E, Ojima H, Kosuge T, Kondo Y, Kanai Y: Carcinogenetic risk estimation based on quantification of DNA methylation levels in liver tissue at the precancerous stage. Int J Cancer 2011;129:11701179.

26 Shen J, Wang S, Zhang YJ, Kappil M, Wu HC, Kibriya MG, Wang Q, Jasmine F, Ahsan H, Lee PH, Yu MW, Chen CJ, Santella RM: Genome-wide DNA methylation profiles in hepatocellular carcinoma. Hepatology 2012;55:1799-1808. 
-27 Shen YC, Lin ZZ, Hsu CH, Hsu C, Shao YY, Cheng AL: Clinical trials in hepatocellular carcinoma: An update. Liver Cancer 2013;2:345-364.

28 Nishida N, Kudo M: Recent advancements in comprehensive genetic analyses for human hepatocellular carcinoma. Oncology 2013;84(Suppl 1):93-97.

29 Kudo M: Why does every hepatocellular carcinoma clinical trial using molecular targeted agents fail? Liver Cancer 2012;1:59-60.

-30 Silverman LR, Demakos EP, Peterson BL, Kornblith AB, Holland JC, Odchimar-Reissig R, Stone RM, Nelson D, Powell BL, DeCastro CM, Ellerton J, Larson RA, Schiffer CA, Holland JF: Randomized controlled trial of azacitidine in patients with the myelodysplastic syndrome: a study of the cancer and leukemia group B. J Clin Oncol 2002;20:2429-2440.

-31 Kantarjian H, Issa JP, Rosenfeld CS, Bennett JM, Albitar M, DiPersio J, Klimek V, Slack J, de Castro C, Ravandi F, Helmer R 3rd, Shen L, Nimer SD, Leavitt R, Raza A, Saba H: Decitabine improves patient outcomes in myelodysplastic syndromes: results of a phase III randomized study. Cancer 2006;106:1794-1803.

32 Goffin J, Eisenhauer E: DNA methyltransferase inhibitors - state of the art. Ann Oncol 2002;13:1699-1716.

33 Dokmanovic M, Clarke C, Marks PA: Histone deacetylase inhibitors: overview and perspectives. Mol Cancer Res 2007;5:981-989.

34 Snykers S, Henkens T, De Rop E, Vinken M, Fraczek J, De Kock J, De Prins E, Geerts A, Rogiers V, Vanhaecke T: Role of epigenetics in liver-specific gene transcription, hepatocyte differentiation and stem cell reprogrammation. J Hepatol 2009;51:187-211.

-35 Lachenmayer A, Toffanin S, Cabellos L, Alsinet C, Hoshida Y, Villanueva A, Minguez B, Tsai HW, Ward SC, Thung S, Friedman SL, Llovet JM: Combination therapy for hepatocellular carcinoma: additive preclinical efficacy of the HDAC inhibitor panobinostat with sorafenib. J Hepatol 2012;56:1343-1350.

-36 Wu LM, Yang Z, Zhou L, Zhang F, Xie HY, Feng XW, Wu J, Zheng SS: Identification of histone deacetylase 3 as a biomarker for tumor recurrence following liver transplantation in HBV-associated hepatocellular carcinoma. PLoS ONE 2010;5:e14460.

-37 Song P, Gao J, Inagaki Y, Kokudo N, Hasegawa K, Sugawara Y, Tang W: Biomarkers: evaluation of screening for and early diagnosis of hepatocellular carcinoma in Japan and China. Liver Cancer 2013;2:31-39. 